\title{
Patterned Self-Assembled Monolayers for Bottom-Up Fabrication of Nanoarchitectures
}

\author{
Chen Fang* \\ Energy Storage and Distributed Resources Division, Lawrence Berkeley National Laboratory, Berkeley, \\ California 94720, the United States \\ Email: cfang@lbl.gov (C.F.)
}

\begin{abstract}
Molecular self-assemblies on surfaces and their bottom-up fabrication applications have been key topics in the field of nanotechnology. Impressively complicated self-assembled monolayers (SAMs) with well-defined nanopatterns have been created on surface employing sophisticated organic molecules. Nevertheless, very limited progress has been made toward the ultimate objective, bottom-up fabrication of nanoarchitectures. This perspective aims to summarize recent advances of patterned SAMs, to identify the obstacles, and also to explore promising directions that will facilitate the development and application of SAMs.
\end{abstract}

Keywords self-assembled monolayers, bottom-up, scanning probe microscopy, MALDI-TOF-MS, surface chemistry

In the past few decades, surfaces and interfaces have been crucial topics for nanotechnology. They not only offer the opportunity to advance our understanding about interactions of matters at molecular and even atomic level, but also serve as platforms for construction of nanoscale features. ${ }^{[1]}$ Scanning tunneling microscopy (STM) has been widely employed for imaging featured surfaces with sub-nanometer resolution and has facilitated rapid advances of surface-based nanotechnology. ${ }^{[2]}$ Patterned self-assembled monolayers (SAMs) are molecular networks physisorbed on surfaces with well-defined nanoscale configuration and periodicity, commonly fabricated via spontaneous assembly of molecules at solid-liquid interface ${ }^{[3]} \mathrm{A}$ broad range of rational designs of ordered SAMs have been proposed and verified with extensive STM studies. However, there have been few reports on the eventual bottom-up fabrication application, which is the ultimate objective for development of SAMs. This perspective serves to provide a brief overview of the key achievements of the field in the past few years, by which the main obstacles and promising future directions are identified.

\section{Surface Nanopatterning with SAMs}

Self-assembly is a convenient and fascinating tool for generation of highly complex networks with simple components. Shang et al. ${ }^{[4]}$ reported the fabrication of molecular Sierpiński triangles on surface with two $\mathrm{V}$-shaped small organic molecules, 4,4"-dibromo-1,1':3',1"'-terphenyl (B3PB) and 4,4"'-dibromo$1,1^{\prime}: 3^{\prime}, 1^{\prime \prime}: 4 ", 1$ '"'-quaterphenyl (B4PB) (Figure 1a). Both molecules possess $120^{\circ}$-bent backbones, which could align with each other in a head-to-tail manner to produce the Sierpiński triangles (Figures $1 \mathrm{~b}$ and $1 \mathrm{c}$ ). It should be noted that these Sierpiński triangles were produced by vapor phase deposition of the molecules on $\mathrm{Ag}(111)$ surface under high vacuum condition, which is a disadvantage for large-scale production.

In recent years, there has been a trend to fabricate complex SAMs at solid-liquid interfaces under ambient condition by simple dropcast method. ${ }^{[1-3]}$ The most successful approaches for SAM formation are host-guest strategy and shape selection strategy. Host-guest strategy aims to create porous 2D net- works on surface to accommodate functionalities. On the other hand, shape selection strategy emphasizes patterned dense packing of molecules strictly dictated by their shapes. Velpula et al. ${ }^{[5]}$ reported a four component host-guest network on highly oriented pyrolytic graphite (HOPG), where the guest coronene molecules were hosted by one shell of isophthalic acid molecules and two shells of differently sized dehydrobenzo[12]annulene molecules. Nevertheless, the uniformity of the SAMs was rather limited due to the lack of molecular selectivity. a

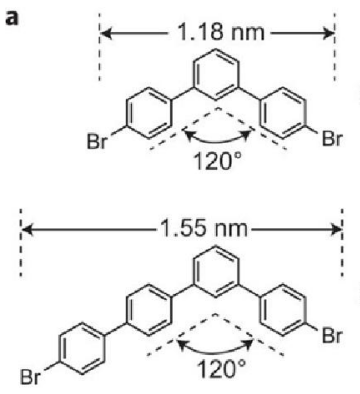

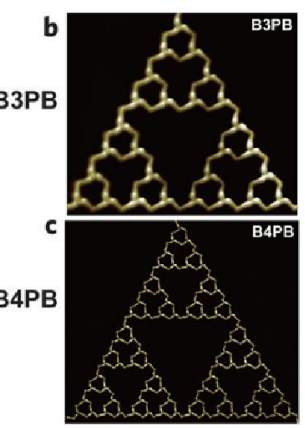

Figure 1 (a) B3PB and B4PB molecules. STM images of Sierpiński triangles of (b) B3PB molecules and (c) B4PB molecules. Adapted from Ref. [4] by permission from Springer Nature, Copyright 2015.

Xue et al. ${ }^{[6]}$ developed an alternative four-component design based on shape selection principle. Four anthracene molecules with shape-complementary alkadiyne chains could orderly pair with their designated neighbors to produce uniform four-component SAMs over a few hundred nanometers on the HOPG substrate. This work demonstrates the importance of strict shape selection for creation of long-range ordered SAMs.

\section{Obstacles for Development of Patterned SAMs}

Despite the encouraging developments of patterned SAMs, the progress on the ultimate application of bottom-up fabrication is limited. A few obstacles are discussed in this section.

Firstly, bottom-up fabrication demands extension of the 


\section{Perspective}

SAMs from the assembly plane (e.g., HOPG surface) into the $3 \mathrm{D}$ space. From a molecular design viewpoint, the out-of-plane moieties are unavoidably flexible and mobile within the allowed range. However, STM is not suitable for imaging such movable features. Du et al. ${ }^{[7]}$ reported the first example of robust fullerene nanoarchitectures covalently templated by the underlying Janus tecton SAMs. The authors found that the flexibility of the fullerenes poses a major challenge for STM imaging. Such an observation is consistent with other recent reports, where highly flexible out-of-plane units of the SAMs could not be resolved by STM and may in turn interfere with the imaging of in-plane structures. ${ }^{[8,9]}$

Secondly, the assembly of multi-component SAMs may require careful tuning of the fabrication process such as the ratio of the components in the dropcast solution. ${ }^{[9]}$ Otherwise, undesired defects could dominate the SAMs. ${ }^{[5,9]}$ STM could in principle be used to screen the SAM formation conditions, but practically speaking, it may be inapplicable as STM measurement is generally rather time-consuming at ambient condition.

Thirdly, bottom-up fabrication demands surface reactions for installation of various functionalities onto the SAMs, which is crucial for the versality of SAMs. ${ }^{[8,9]}$ There have been few reports exploring SAM reactions because the contaminations left by the surface chemistry, even trace amount, could significantly reduce the resolution of the STM images and even prohibit effective imaging. ${ }^{[9]}$

Recently, mass spectrometry (MS) has been applied for characterization of pattern SAMs on HOPG ${ }^{[8,9]}$ MS would not be affected by the mobile components of the SAMs and would not be strongly interfered with by the residue chemicals from surface reactions. In addition, MS is capable of fast screening of SAMs produced under various conditions (few minutes per sample) and thus can quickly optimize assembly parameters.

Separately, the reactions of patterned SAMs physisorbed on surfaces are rather challenging. Patterned SAMs rely on physisorption, a reversable process, to correct the "assembly errors" (i.e., morphology defects) by equilibrium between adsorption and desorption. Therefore, the SAMs are only weakly bonded to the substrate and could be readily rinsed off upon contact with many common organic solvents. Only few solvents (e.g., tetradecane and dimethylformamide) have been demonstrated to be suitable for SAM reactions. ${ }^{[8,9]}$ In addition, the existence of residue reagents, catalysts and/or by-products on SAMs is inevitable, and therefore, surface rinsing is a necessary work-up step but needs to be carried out with great caution to avoid desorption of the weakly adsorbed SAMs.

The next section demonstrates a new surface reaction philosophy of "functionalization after stabilization" and describes the MS methods for SAM characterization.

\section{Mass Spectrometry and Surface Reactions for SAMs}

Matrix-assisted laser desorption ionization-time of flight mass spectrometry (MALDI-TOF-MS) has been applied to characterize chemisorbed monolayers as well as their chemical transformations.$^{[10,11]}$ MALDI is intrinsically suitable for analyzing the chemical species on surfaces. With a modified MALDI plate that can accommodate a HOPG-holding metal case, MALDI-TOF-MS has been proven compatible for physisorbed SAMs on graphite surface as well. ${ }^{[8]}$ The universality of the methodology on different molecular species has also been confirmed. ${ }^{[8,9,12]}$

In a recent report, ${ }^{[9]}$ the covalent bottom-up fabrication of high-complexity nanoarchitectures by SAM templates with surface reactions has been demonstrated for the first time. This system is distinct from other previously reported nanostructures that were merely based on weak non-covalent interactions. ${ }^{[13-15]}$ Two triphenylene ethynylene (TPE) molecules $1^{\mathrm{OH}}$ and 2 were synthesized for creation of patterned SAMs via shape selection of alkadiyne chains (Figure 2a). These two TPE molecules individually close-pack into columns on HOPG surface with alternating composition of $\mathbf{1}^{\mathrm{OH}}$ and $\mathbf{2}$ in the neighboring columns (molar ratio $1: 1$ ). MALDI assisted to discover that excess 2 molecules were needed to yield the desired 1:1 SAMs due to their lower affinity to the surface. Nextly, surface dimerization reaction of $1^{\mathrm{OH}}$ with a disulfide linker stabilized the SAM system and provided anchoring point for gold nanoparticles (AuNPs), by which the features of SAMs were translated into the patterns of captured AuNPs on surface (Figures $2 b$ and 2c, bright features correspond to the AuNPs). This dimerization reaction was monitored and optimized by MALDI since STM was incapable of verifying such chemical transformations. It is worth noting that the patterned AuNP nanostructures were found to be uniform over nearly $1 \mu \mathrm{m}$, almost reaching beyond the boundary of "nano world". The application of MALDI for fast SAM characterization and surface reaction analysis was essential for the success of this work (MALDI spectrum of $1: 1$ $1^{\mathrm{OH}}: 2$ SAMs shown in Figure 2d).
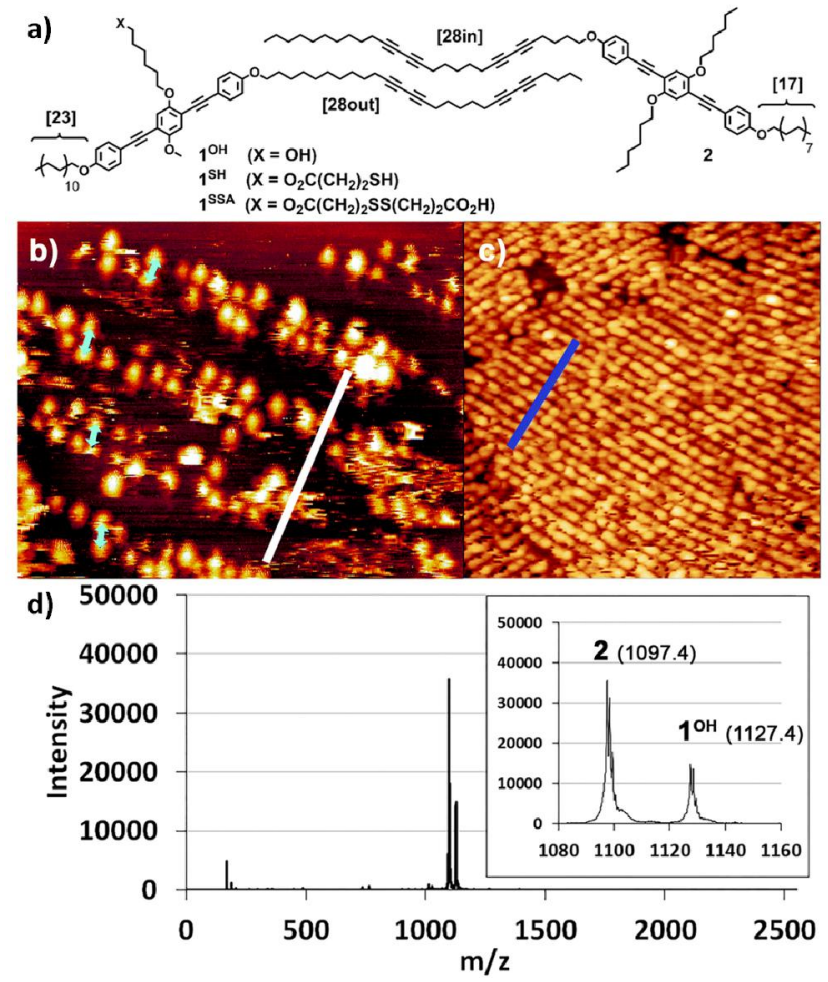

Figure 2 (a) TPE molecule $1^{\mathrm{OH}}$ and 2. (b) STM image (100 $\times$ $75 \mathrm{~nm})$ and (c) AFM image $(600 \times 600 \mathrm{~nm})$ of patterned AuNP surface. (d) MALDI spectrum of $1^{\mathrm{OH}}: 2$ SAMs with $1: 1$ molar ratio. Adapted from Ref. [9] with permission from The Royal Society of Chemistry, Copyright 2018.

\section{Conclusion and Outlook}

SAMs are versatile and facile platforms for construction of sophisticated nanoarchitectures with precise molecular control and tunable periodicity. The patterned SAMs could serve as nano-templates for complex bottom-up fabrication applications when combined with surface chemistry. STM characterization of SAMs has various limitations as STM is not suitable for 3D structures or contaminated surfaces. MALDI-TOF-MS is a high-throughput, surface-compatible analytical tool that could 
readily analyze SAMs that are inapplicable for classic STM experiments. Separately, due to the weak interactions between SAMs and the substrate, surface reactions need to be designed carefully to avoid desorption of the SAMs. Crosslinking of the SAM components before further surface reactions is an effective strategy for improving their robustness. The combination of MALDI and SAM-specific surface reactions will inspire further renovations in bottom-up fabrication of highly ordered nanoarchitectures with SAM nano-templates.

\section{References}

[1] Cui, D.; MacLeod, J. M.; Rosei, F. Chem. Commun. 2018, 54, 10527.

[2] Goronzy, D. P.; Ebrahimi, M.; Rosei, F.; Arramel, Fang, Y.; De Feyter, S.; Tait, S. L.; Wang, C.; Beton, P. H.; Wee, A. T.; Weiss, P. S. ACS Nano 2018, 12, 7445.

[3] Mali, K. S.; Pearce, N.; De Feyter, S. Champness, N. R. Chem. Soc. Rev. 2017, 46, 2520.

[4] Shang, J.; Wang, Y.; Chen, M.; Dai, J.; Zhou, X.; Kuttner, J.; Hilt, G.; Shao, X.; Gottfried, J. M.; Wu, K. Nat. Chem. 2015, 7, 389.

[5] Velpula, G.; Takeda, T.; Adisoejoso, J.; Inukai, K.; Tahara, K.; Mali, K. S.; Tobe, Y.; De Feyter, S. Chem. Commun. 2017, 53, 1108.

[6] Xue, Y.; Zimmt, M. B. J. Am. Chem. Soc. 2012, 134, 4513.
[7] Du, P.; Kreher, D.; Mathevet, F.; Maldivi, P.; Charra, F.; Attias, A. J. ChemPhysChem 2015, 16, 3774.

[8] He, J.; Fang, C.; Shelp, R. A.; Zimmt, M. B. Langmuir 2016, 33, 459.

[9] Fang, C.; Zhu, H.; Chen, O.; Zimmt, M. B. Chem. Commun. 2018, $54,8056$.

[10] Houseman, B. T.; Gawalt, E. S.; Mrksich, M. Langmuir 2003, 19 , 1522.

[11] Montavon, T. J.; Li, J.; Cabrera-Pardo, J. R.; Mrksich, M.; Kozmin, S. A. Nat. Chem. 2012, 4, 45.

[12] Cheng, K. Y.; Lee, S. L.; Kuo, T. Y.; Lin, C. H.; Chen, Y. C.; Kuo, T. H.; Hsu, C. C.; Chen, C. H. Langmuir 2018, 34, 5416.

[13] Mezour, M. A.; Perepichka, I. I.; Zhu, J.; Lennox, R. B.; Perepichka, D. F. ACS nano 2014, 8, 2214.

[14] Cui, D.; MacLeod, J. M.; Ebrahimi, M.; Perepichka, D. F.; Rosei, F. Chem. Commun. 2015, 51, 16510.

[15] González, J. D. C.; lyoda, M.; Rabe, J. P. Nat. Commun. 2017, 8, 14717.

Received August 14, 2019 Accepted September 5, 2019 